\title{
Hyperpigmentation, CTCAE
}

National Cancer Institute

\section{Source}

National Cancer Institute. Hyperpigmentation, CT CAE. NCI Thesaurus. Code C57998.

An adverse reaction characterized by darkening of the skin. 